\title{
Preoperative fasting times: Prescribed and actual fasting times at Universitas Hospital Annex, Bloemfontein, South Africa
}

\author{
G Lamacraft, ${ }^{1}$ MBBS (Lond), DA (SA), MRCP (UK), FRCA (UK), PhD; C Labuschagne, ${ }^{2}$ 3rd-year medical student; \\ S Pretorius, ${ }^{2}$ 3rd-year medical student; M C Prinsloo, ${ }^{2}$ 3rd-year medical student; M D Smit, ${ }^{2}$ rd-year medical student; \\ J-R Steyn, ${ }^{2}$ 3rd-year medical student \\ ${ }^{1}$ Department of Anaesthesiology, Faculty of Health Sciences, University of the Free State, Bloemfontein, South Africa \\ ${ }^{2}$ School of Medicine, Faculty of Health Sciences, University of the Free State, Bloemfontein, South Africa
}

Corresponding author: G Lamacraft (lamacraftg@ufs.ac.za)

\begin{abstract}
Background. Guidelines recommend a preoperative fasting period of 6 hours for solid food and 2 hours for clear fluids. Because of fixed meal times and imprecise operation starting times, patients often fast for an extended period of time.

Objective. To investigate the prescribed preoperative fasting times, and the actual duration of fasting, compared with the internationally accepted fasting times for solid food and clear fluids.

Methods. Patients $(N=105)$ aged 14 - 60 years, who were scheduled for elective surgery in the morning session (list starting time 07h00), were included in this prospective study. On arrival in theatre, all patients were asked when they last ate and drank. Anaesthetic records were used to determine the prescribed fasting times and operation starting times.

Results. For solids, patients were most frequently prescribed to start fasting from $22 \mathrm{~h} 00$ to $00 \mathrm{~h} 00$ ( $53.3 \%$ and $39.1 \%$, respectively). No patient fasted $<8$ hours. The median duration of fasting was 14 hours and 45 minutes (range 9 hours and 45 minutes - 19 hours and 5 minutes). For fluids, patients were most frequently prescribed to start fasting from $05 \mathrm{~h} 00(46.7 \%), 00 \mathrm{~h} 00(27.6 \%)$ and $22 \mathrm{~h} 00$ (7.6\%). In practice, no patient ingested fluids after $22 \mathrm{~h} 30$ or $<9$ hours preoperatively. The median fasting time for oral fluids was 13 hours and 25 minutes (range 9 hours and 37 minutes - 19 hours and 5 minutes).

Conclusion. Most patients started fasting too early preoperatively, consequently withholding food and oral fluids for longer than recommended. An increased awareness regarding complications of unnecessarily long fasting times, and interventions to correct this problem, is required.
\end{abstract}

S Afr Med J 2017;107(10):910-914. DOI:10.7196/SAMJ.2017.v107i10.10930

The current international guidelines for preoperative fasting for elective surgery are 6 hours for solid food and 2 hours for clear oral fluids. ${ }^{[1]}$ The guidelines are based on evidence that these time periods are required for gastric emptying to be complete. Patients fasting for shorter periods of time are at risk of having residual contents in their stomach, which may be aspirated during anaesthesia. ${ }^{[2]}$

Conversely, if patients fast for too long, it is associated with problems, including increased preoperative nausea and vomiting, and postoperative delayed awakening from hypoglycaemia, particularly in neonates. ${ }^{[3,4]}$ Tissue repair is impaired by dehydration, and wound healing is improved when correct fluid fasting times are implemented. ${ }^{[5]}$ To ameliorate these problems, the administration of carbohydrate-rich, clear oral fluids 2 hours preoperatively has been advocated. ${ }^{[6]}$

There are several reasons why preoperative fasting periods may be too long or too short. Doctors may not be up to date with the recommended guidelines, and some hospitals have policies on fasting that differ from the recommended guidelines. ${ }^{[7]}$ However, it is the policy of the Department of Anaesthesiology at the University of the Free State (UFS), Bloemfontein, South Africa (SA), to adhere to the international guidelines mentioned above. Our study was performed to determine whether this policy was being followed and patients were being starved correctly preoperatively.

The Universitas Hospital Annex in Bloemfontein was chosen as the site for this study, as it is a relatively small operating complex where elective orthopaedic and ophthalmic surgery is performed on weekday mornings in three operating theatres (emergency cases are done in the afternoons). The theatre lists at the Annex are less often interrupted by emergencies, as is the case at the busier academic hospitals in Bloemfontein, and the Annex therefore should be the environment providing the best conditions for adherence to correct fasting times.

\section{Methods}

This prospective study was performed from 18 June to 30 September 2012. Patients included those scheduled for elective ophthalmic surgery under general anaesthesia or orthopaedic surgery under regional or general anaesthesia during the morning sessions at the Universitas Hospital Annex theatre complex.

Exclusion criteria included patients $<14$ or $>60$ years of age, and those who, on arrival in theatre, could not indicate when they last ate or drank, e.g. because of sedative effects of premedication or language difficulties. Patients undergoing ophthalmic surgery under regional anaesthesia were excluded, as they do not routinely fast.

It is standard practice for the receiving theatre nursing staff to ask patients arriving at the Annex theatre complex when they last ate or drank, which is recorded in the nursing file. For the purpose of this study, the receiving theatre nurse also recorded this information on a separate research form, as well as whether an intravenous (IV) infusion of fluid was in situ. The anaesthesiologist placed a copy of the anaesthesiology record form of each patient in a collecting box in theatre at the end of each procedure. The researchers collected the anaesthesia and nursing forms on a weekly basis and recorded the relevant information, which included patient demographics, operation starting time, time preoperative fasting of solid foods and clear fluids was prescribed to commence, and time the patient last 
ingested food or clear fluids preoperatively. The data were analysed by the Department of Biostatistics, UFS. The data were categorised and frequencies and percentages of the categories were calculated. The results of the fasting times were compared with the recommended preoperative fasting guidelines. ${ }^{[1]}$

\section{Ethical appoval}

Research approval (ref. no. STUD 14/2012) was obtained from the Ethics Committee of the Faculty of Health Sciences, UFS.

\section{Results}

During the research period, 249 patients underwent surgery, of whom 105 were eligible for inclusion in the study. The mean age of the patients was 36 (range 14 - 60) years; standard deviation (SD) 14.4). Males comprised 62.9\% of the study patients. Six (5.7\%) patients arrived at the theatre with a running IV fluid infusion.

\section{Operation starting times}

Operations started between $07 \mathrm{~h} 00$ and $12 \mathrm{~h} 30$ - 13 h00 (Table 1). One procedure started early, at $06 \mathrm{~h} 45$.

\section{Prescribing of fasting times}

Most patients were prescribed to start fasting from solids either from $22 \mathrm{~h} 00$ ( $n=56 ; 53.3 \%)$ or midnight $(n=40 ; 39.1 \%)$. A small number of patients $(n=9 ; 8.6 \%)$ were prescribed to start fasting from solids at any other time. For fluids, the starting times for fasting were $05 \mathrm{~h} 00$ $(n=49 ; 46.7 \%)$, midnight $(n=29 ; 27.6 \%)$ and $22 \mathrm{~h} 00$ ( $n=8 ; 7.6 \%)$. The remaining $19(18.1 \%)$ patients were prescribed to start fasting from fluids at other times.

\section{Actual times patients ate or drank preoperatively}

Patients most frequently ingested their last solid food preoperatively at $17 \mathrm{~h} 00$ or $20 \mathrm{~h} 00$ (31.4\% and $29.5 \%$, respectively). The earliest

Table 1. Operation starting times $(N=105)$

\begin{tabular}{ll}
\hline Time & $\boldsymbol{n}(\%)$ \\
\hline 06h00 - 06h59 & $1(1.0)$ \\
07h00 - 07h29 & $4(3.8)$ \\
07h30 - 07h59 & $19(18.1)$ \\
08h00 - 08h29 & $8(7.6)$ \\
08h30 - 08h59 & $7(6.7)$ \\
09h00 - 09h29 & $12(11.4)$ \\
09h30 - 09h59 & $13(12.4)$ \\
10h00 - 10h29 & $7(6.7)$ \\
10h30 - 10h59 & $13(12.4)$ \\
11h00 - 11h29 & $8(7.6)$ \\
11h30 - 11h59 & $7(6.7)$ \\
12h00 - 12h29 & $5(4.8)$ \\
12h30 - 13h00 & $1(1.0)$
\end{tabular}

time patients ingested solids preoperatively was $16 \mathrm{~h} 00(n=2 ; 1.9 \%)$ and the latest was $22 \mathrm{~h} 00$ ( $n=4 ; 3.8 \%$ ) (Fig. 1).

With regard to liquids, the most frequent times patients last ingested fluids orally were $20 \mathrm{~h} 00$ ( $n=39 ; 37.1 \%), 21 \mathrm{~h} 00(n=29 ; 27.6 \%)$ and $22 \mathrm{~h} 00$ ( $n=12 ; 11.4 \%)$. The earliest time patients stopped drinking was $16 \mathrm{~h} 00(n=2 ; 1.9 \%)$, and the latest time was $22 \mathrm{~h} 30$ ( $n=1 ; 1.0 \%)$ (Fig. 2$)$.

\section{Comparison of prescribed v. actual fasting times}

Only $2(1.9 \%)$ patients fasted from solids from the time prescribed. The median time that patients fasted longer than prescribed was 5 hours and 42 minutes ( 1 hour and 40 minutes - 17 hours and 35 minutes).

More than a quarter $(n=28 ; 26.8 \%)$ of patients fasted from food $\geq 2$ hours than prescribed, and 32 (30.6\%) for $\geq 5$ hours than prescribed (Table 2). One patient fasted an hour shorter than the time prescribed by the doctor, but still fasted $>7$ hours. No patient fasted from food for $<6$ hours or fluid $<2$ hours.

When comparing the prescribed and actual times for fasting from the intake of clear fluids, $42.3 \%$ of patients did not ingest fluids for at least 8 hours longer than prescribed. One patient ingested no oral fluids for 16 hours preoperatively. Only three (2.9\%) patients ingested clear fluids 2 hours preoperatively (Table 2 ).

We calculated the time difference between the fasting guidelines and the prescribed fasting times. Only 1 patient was correctly prescribed to fast from solid food for 6 hours before the operation started, while 91 (86.7\%) were instructed to fast at least 2 hours longer than this recommended fasting time. There were 2 patients who were prescribed to fast for

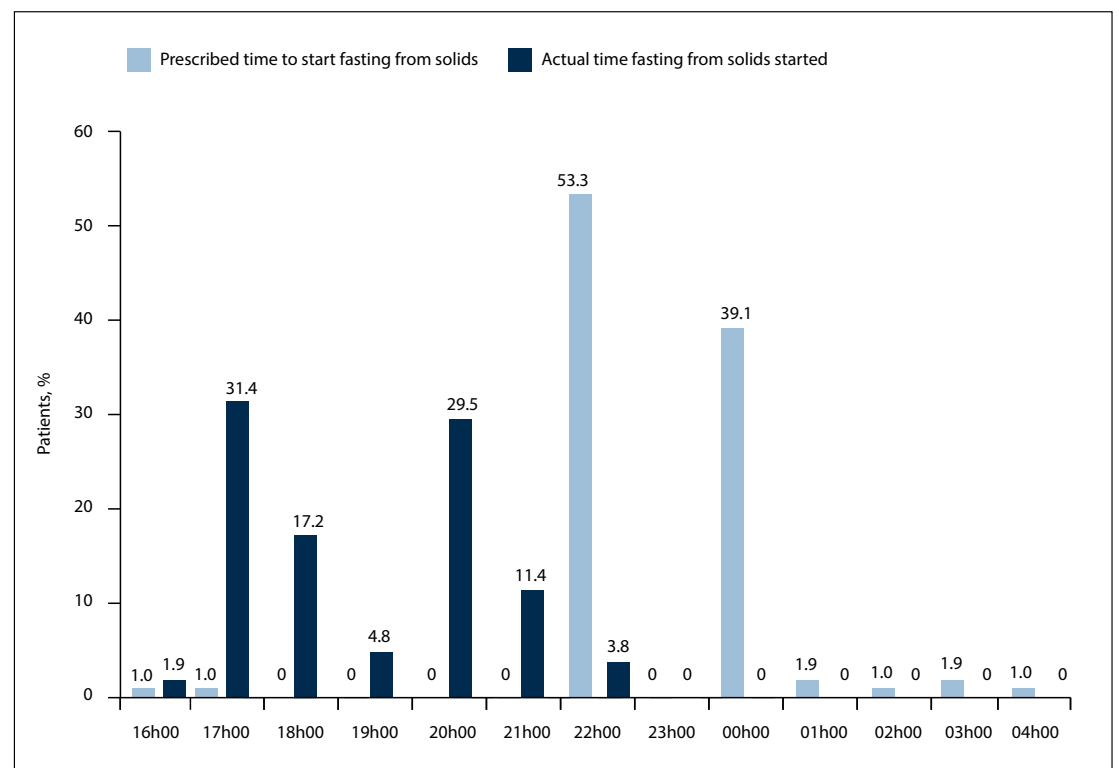

Fig. 1. Actual time that patients started fasting from solid food compared with the time prescribed by the doctor.

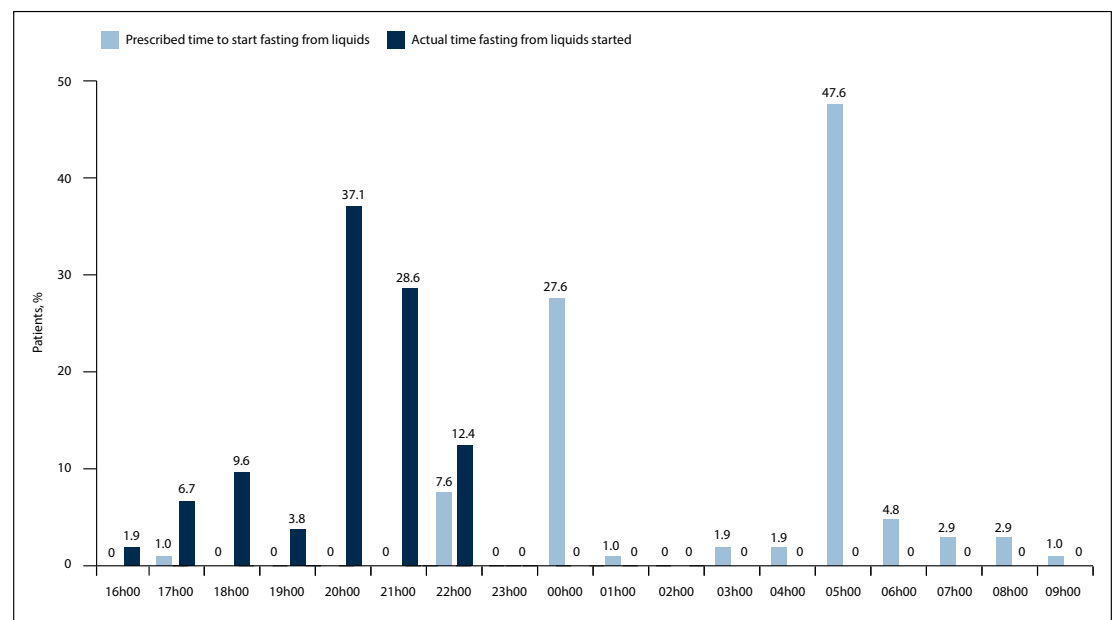

Fig. 2. Actual time that patients started fasting from liquids compared with the time prescribed by the doctor. 
$\geq 11$ hours than stipulated by the guidelines (Fig. 3).

For clear fluids, 20 (19.0\%) patients were correctly prescribed to fast for only 2 hours preoperatively, and $72(68.8 \%)$ for $\geq 2$ hours than the international guideline for fasting from clear fluids (Fig. 4).

The duration from the start of fasting from solid food until the operation commenced is shown in Fig. 5. No patient ingested solid food $<9$ hours before an operation. The mean duration of fasting from solid food was 10 hours. Most patients last ingested solid food 13 14 hours preoperatively, with the longest time 19 - 20 hours. Similarly, no patients ingested fluids $<6$ hours preoperatively. The mean duration of time that fluids had not been ingested preoperatively was 13 hours ( 9 hours and 37 minutes - 19 hours and 5 minutes) (Fig. 6).

\section{Discussion}

Our findings showed that patients frequently fasted for longer periods than prescribed. On average, patients fasted 4 hours longer than the international guidelines for solid food and 8 hours longer than internationally prescribed for clear oral fluids. Furthermore, $<6 \%$ of patients kept to the doctor's fasting recommendations. Only 1 participant (1.0\%) fasted for a shorter time than prescribed. Approximately $72 \%$ of participants fasted from solid food $2-5$ hours longer than the doctor's recommendation. Almost a quarter (22.9\%) of the patients fasted from clear fluids for 9 hours longer than the doctor prescribed.

In previous years, the routine at Universitas Academic Hospital was for preoperative patients to fast from $22 \mathrm{~h} 00$ or midnight with regard to both food and fluids. However, the recent literature ${ }^{[1,3,8]}$ demonstrated that prolonged fasting could be detrimental to a patient's welfare and wound healing. Therefore, the Department of Anaesthesiology changed its practice regarding preoperative fasting times more than a decade ago and has since taught medical students and anaesthesiology registrars to use the fasting guidelines of 6 hours for solid food and 2 hours for clear fluids.

Despite this teaching, our study showed that the former practice of fasting patients from either $22 \mathrm{~h} 00$ or midnight remains prevalent, although theatre lists rarely start before $07 \mathrm{~h} 30$. This pattern of fasting from $22 \mathrm{~h} 00$ and midnight was particularly seen for fasting from solids. It was found - to a lesser extent - that $05 \mathrm{~h} 00$ was the most frequent time to start fasting from clear fluids. These findings show that most doctors who

$\begin{aligned} & \text { Table 2. Time (hours) that patients deviated from doctors' } \\
& \text { times }(\boldsymbol{N}=\mathbf{1 0 5})\end{aligned}$
\begin{tabular}{lll} 
Time deviated, $\boldsymbol{h}$ & Clear fluids, $\boldsymbol{n}$ (\%) & Solid foods, $\boldsymbol{n}$ (\%) \\
\hline$<1^{*}$ & $0(0)$ & $1(1.0)$ \\
0 & $3(2.9)$ & $3(2.9)$ \\
1 & $4(3.8)$ & $4(3.8)$ \\
2 & $2(1.9)$ & $20(19.1)$ \\
3 & $10(9.5)$ & $10(9.5)$ \\
4 & $13(12.4)$ & $21(20.0)$ \\
5 & $3(2.7)$ & $24(22.9)$ \\
6 & $4(3.9)$ & $8(7.6)$ \\
7 & $7(6.7)$ & $9(8.6)$ \\
8 & $16(15.2)$ & $1(1.0)$ \\
9 & $24(22.9)$ & $2(1.9)$ \\
10 & $5(4.8)$ & $1(1.0)$ \\
11 & $5(4.8)$ & $1(1.0)$ \\
12 & $5(4.8)$ & $0(0)$ \\
13 & $3(2.9)$ & $0(0)$ \\
$>13$ & $1(1.0)$ & $0(0)$ \\
$*$ One patient consumed solid food within an hour after fasting should have commenced.
\end{tabular}

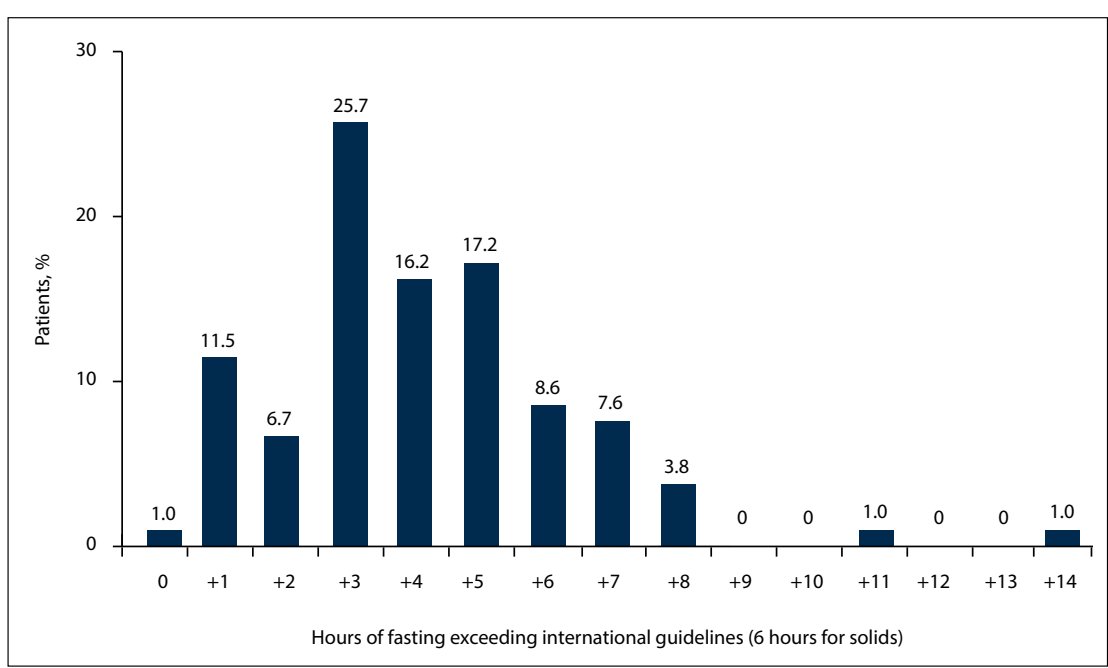

Fig. 3. Time difference between international guidelines for fasting from solid food (6 hours) and duration of patients' fast.

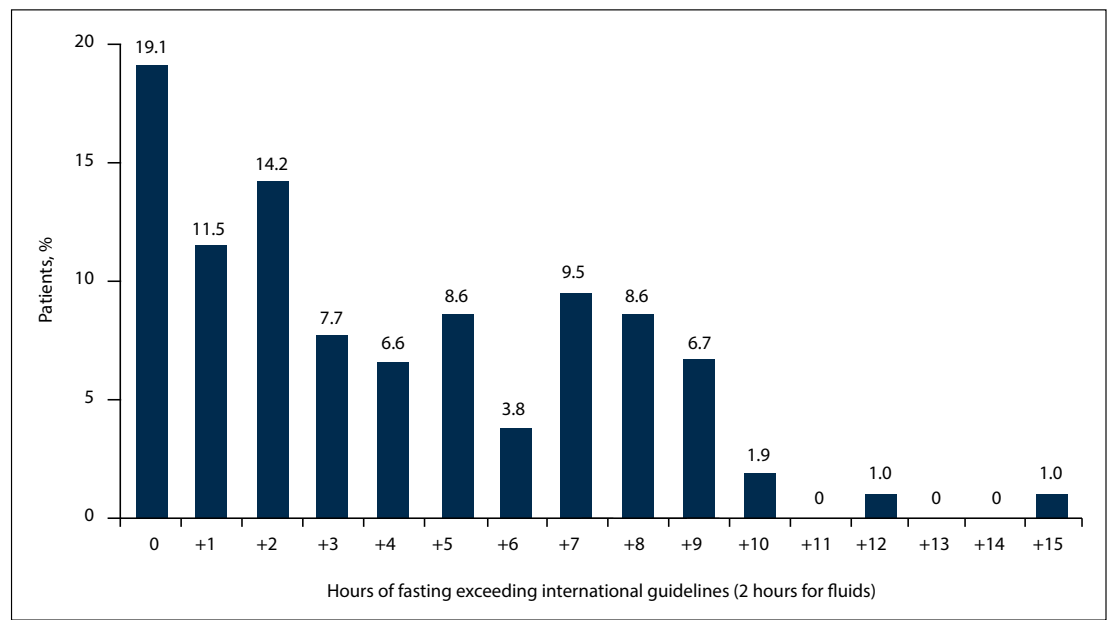

Fig. 4. Time difference between international guidelines for fasting from liquids (2 hours) and duration of patients' fast. 




Fig. 5. Duration of time from start of fasting from solid food and commencement of surgical procedure.

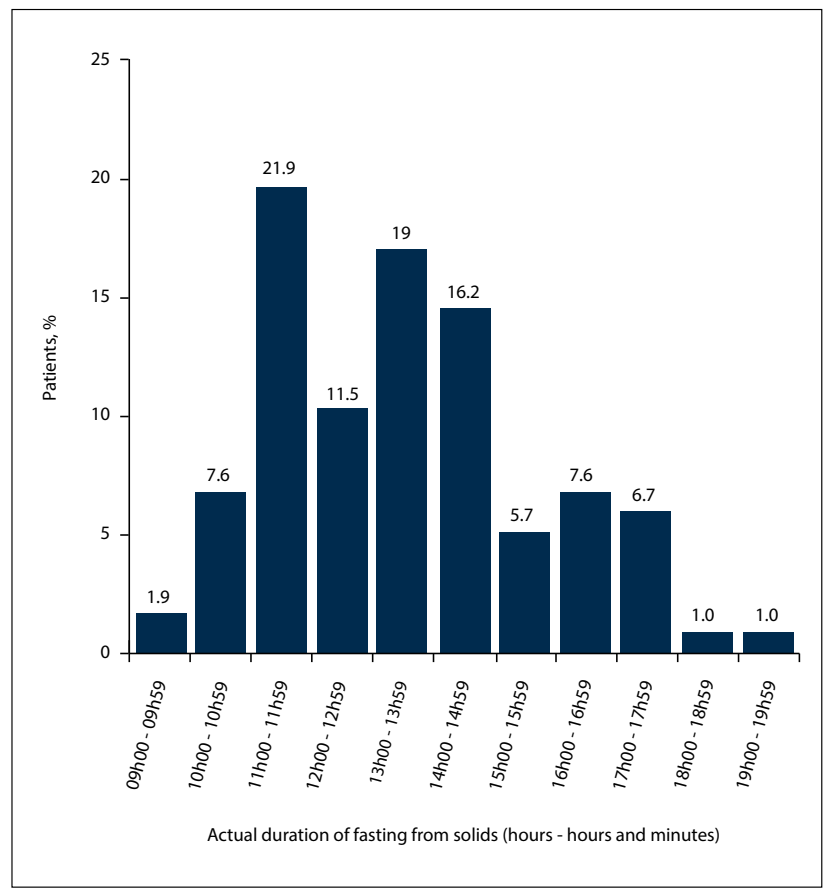

Fig. 6. Duration of time from start of fasting from liquids and commencement of surgical procedure.

prescribe fasting times for patients need further education regarding the correct periods of time that patients should fast.

Some patients do not understand the importance of instructions regarding preoperative fasting, leading to non-compliance with regulations. A study similar to ours measured the effect of patients' understanding of fasting protocols; knowledge and understanding of guidelines were associated with patients showing increased compliance with fasting regulations. ${ }^{[9]}$ Patients and healthcare workers must have sufficient knowledge of preoperative fasting. They must know the importance, as well as the effects and complications, of not complying with these guidelines.

Doctors must correctly prescribe the preoperative fasting times. In our study, operations normally started at $07 \mathrm{~h} 30$. To prevent understarving of patients, doctors can safely prescribe fasting from clear fluids at $05 \mathrm{~h} 00$ and solid food at $01 \mathrm{~h} 00$, which corresponds with the internationally prescribed preoperative fasting guidelines.

The time of the first operation on a theatre list can be predicted with reasonable certainty, although it is not often certain when the following operations on the list will start, as the duration of an operation cannot be accurately predicted. However, the usual theatre times for various types of operations can be analysed and a reasonable estimate of the duration of an operation can be made. From this information, an estimate of the starting time of an operation can be made for subsequent cases on a theatre list. This should be the practice when compiling a theatre list, as it will then not be over- or under-booked. The anaesthesiologist can use the estimated theatre starting time written on the operating list as a guideline for the fasting times for a patient. However, it is often the practice that the estimated operation starting time is not written on the theatre list, with the doctor inserting 'to follow' as the starting time.

The problem of not estimating operation starting times is compounded by the unanticipated changes in the list order owing to equipment or staffing problems. Consequently, patients fast for a longer period than required, as they were probably fasted with the assumption that all operations, regardless of the order on the theatre list, may start at any time from $07 \mathrm{~h} 30$ onwards. Without management strategies to prevent these unexpected changes in the order of theatre lists and failure of doctors who compile the lists to estimate operation starting times, patients whose operations start later continue to be starved excessively.

The over-prescribing of fasting times was exacerbated by patients who fasted for longer than prescribed, which resulted in most patients being starved for $>10$ hours from solids and 13 hours from fluids preoperatively. This practice was unlikely to be deliberate, but was most possibly owing to fixed times of ward meals. At the time of this study, the evening meal for diabetic patients was served at $17 \mathrm{~h} 00$, and a drink with a sandwich at $20 \mathrm{~h} 00$. Patients were not offered food or fluids outside of these times, as there was none available on the ward other than their own provisions. Patients were only given a small sip of water with their premedication on the morning of surgery, even if clear fluids were prescribed until 05h00. In some instances, patients may not have been present in the ward when the evening meal was served, as patients occasionally are sent for preoperative radiographs the day before surgery, and may miss their meal before fasting is due to start, prolonging their actual fasting times. Ward staff need to ensure that patients who have to fast, eat and drink adequately prior to the time fasting is initiated.

Delayed wound healing with an increased risk of infection associated with prolonged fasting is a potential complication in such patients. Another problem usually experienced is postoperative hunger and thirst. Some of the patients were scheduled to have arthroplasty operations, and because of knowledge of this problem, it was routine practice at the time of the study for surgeons to commence these patients on IV fluids the night before operation; hence, some patients $(n=5)$ came to theatre with an IV infusion in situ. This was not routine practice for patients undergoing other types of surgery. If patients are given access to clear fluids until 2 hours preoperatively, it should not be necessary to have IV fluid infusions to prevent preoperative dehydration.

As a result of this study, the hospital will in future supply sandwiches for preoperative patients at $22 \mathrm{~h} 00$ the night before surgery and a carbohydrate-rich clear-fluid drink at $05 \mathrm{~h} 00$ on the day of surgery. It is hoped that these measures will prevent patients from fasting for too long preoperatively.

Similar studies have recently been published. Gebremedhn and Nagaratnam ${ }^{[10]}$ conducted a cross-sectional study, where 43 patients 
underwent elective surgery. Their methods and results were similar to ours. The majority of patients (58\%) fasted from solid food for $>12(5$ - 96) hours, although there were no patients in our study who fasted from solid food $>20$ hours. Similarly, patients fasted too long from clear fluids in the Gebremedhn and Nagaratnam study, ${ }^{[10]}$ with most patients (48.8\%) fasting for $>12$ hours. The degree of postoperative hunger and thirst in these patients did not correlate with the duration of fasting, as $51.2 \%$ of patients who fasted from fluids for $\geq 9$ hours did not experience any thirst postoperatively. ${ }^{[10]}$

Furthermore, the methodology of Cestonaro et al. ${ }^{[1]]}$ who conducted a retrospective study in a public university hospital in the south of Brazil, was very similar to ours. Their results showed that the median period of preoperative fasting from solid food was 16 hours and 30 minutes ( 5 hours and 30 minutes - 56 hours and 55 minutes) and from fluids 15 hours and 45 minutes ( 2 hours and 30 minutes - 56 hours and 55 minutes). The fasting period for most patients ranged between 9 hours and 30 minutes and 21 hours and 30 minutes. One patient fasted from solids and liquids for 56 hours and 55 minutes. The hospital where their study was conducted had no clear guidelines, and internationally prescribed guidelines were not followed. In addition, surgeons, nurses and physicians could prescribe the fasting times. ${ }^{[1]}$

\section{Study limitations}

There are limitations to this study with regard to the reliability of the data collected from the patients. They had to remember the time when they last ingested food or drink, which might not be accurate. The decision whether the patient was over-sedated from premedication or mentally competent and understood the questions, was made by the receiving nurse in theatre and might not have been correct. When interpreting the study results, one must take into account that all patient procedures were scheduled for the morning lists and that they were elective patients. Patients on afternoon theatre lists might receive a morning meal and not fast for very long. Conversely, if a patient had not been served a morning meal, they might have had a protracted period of fasting.

In some cases, the decision to operate on an emergency patient may only be made after a morning consultant ward round, and patients may miss their morning meal in anticipation of the decision to operate later during that day. If other emergencies take precedence, the patient may then be rebooked for the following day, already having fasted throughout the day. In situations where operations are frequently postponed, there is a real risk that patients can become severely malnourished and even die as a result of being over-starved preoperatively. ${ }^{[12]}$ Elective operations are not often postponed at this hospital, but emergency surgery at other hospitals are frequently delayed owing to other emergency cases taking precedence; these patients may be at real risk of suffering the consequences of over-fasting.

\section{Conclusion}

The majority of prescribed fasting times were too long for both solids and fluids. Erroneous implementation of these incorrect fasting times exacerbated the problem, as most of the patients fasted even longer than the doctors prescribed.

Doctors who prescribe fasting times need to be aware of the negative impact their incorrect prescriptions have on patients. Nursing staff should endeavour to allow patients access to food and clear fluids until the time from which the patients are prescribed to fast. Consideration should be given to prescribing a clear, carbohydrate- rich drink for patients early in the morning (e.g. at 05h00) to ensure that fasting is not prolonged.

We recommend that the hospital staff should serve a glass of water or a nutritional drink, such as apple juice, on a standard basis at $05 \mathrm{~h} 00$, to all patients awaiting surgery, so that patients do not fast from clear fluids for too long. Such an approach has been proven to have no adverse effect on gastric volume and $\mathrm{pH}$ preoperatively compared with water or fasting from midnight. ${ }^{\left[8_{1,2]}\right.}$ It has the positive effect of decreasing insulin resistance ${ }^{[12,13]}$ and increasing recovery ${ }^{[14]}$ of patients postoperatively. As most operations start after $07 \mathrm{~h} 00$, this drink of water or apple juice falls within the international fasting guideline of 2 hours for clear fluids.

Hospital staff should encourage patients to drink water until the recommended fasting time, so that autonomous patients take care of themselves and prevent over-fasting. Encourage eating, where possible, until midnight.

Acknowledgements. Mr Cornel van Rooyen, Department of Biostatistics, UFS, for assistance with the analysis and interpretation of the data; and Dr Daleen Struwig, Faculty of Health Sciences, UFS, for technical and editorial preparation of the manuscript.

Author contributions. The medical students (CL, SP, MCP, MDS, J-RS) wrote the protocol and collected the data under the guidance of their supervisor, GL. The literature search was done by GL. The article was written by the medical students, with revisions by GL.

Funding. The University of the Free State supported the authors' costs for their transport to the National Hospital, as well as for stationery and printing. No external funding was received.

Conflicts of interest. None.

1. Brady M, Kinn S, Stuart P. Preoperative fasting for adults to prevent perioperative complications Cochrane Database Syst Rev 2003;23(4):CD004423. https://doi.org/10.1002/14651858.CD004423 2. Olsson GL, Hallen B, Hambraeus-Jonzon H. Aspiration during anesthesia: A computer aided study of 185 358 anaesthetics. Acta Anaesthesiol Scand 1986;30(1):84-92. https://doi.org/10.1111/j.1399-6576.1986. tb02373.x

3. Kruger MT, Short TG. Aspiration during anaesthesia: A review of 133 cases from the Australian Anaesthetic Incident Monitoring Study (AIMS). Anaesthesia 1999;54(1):19-26. https://doi.org/10.1046/ j.1365-2044.1999.00642.x

4. O'Hare B, Lerman J, Endo J, Culz E. Acute lung injury after instillation of human breast milk or infant formula into rabbits' lungs. Anaesthesiology 1996;84(6):1386-1391. https://doi.org/10.1097/00000542199606000-0001

5. Brandstrup B, Tønnesen H, Beier-Holgerson R, et al. The effect of intravenous fluid restriction on postoperative complications: Comparison of two perioperative fluid regimens. A randomized assessor-blinded multicenter trial. Ann Surg 2003;238(5):641-648. https://doi.org/10.1097/01. sla.0000094387.50865.23

6. Bannister WK, Sattilaro AJ. Vomiting and aspiration during anesthesia. Anesthesiology 1962;23(2):251-264. https://doi.org/10.1097/00000542-196203000-00012

7. Breuer JP, Bosse G, Seifert S, et al. Pre-operative fasting: A nationwide survey of German anaesthesia departments. Acta Anaestesiol Scand 2010;54(3):313-320. https://doi.org/10.1111/j.1399-6576.2009.02123.x 8. Stuart PC. The evidence base behind modern fasting guidelines. Best Pract Res Clin Anaesthesio 2006;20(3):457-469. https://doi.org/10.1016/j.bpa.2006.03.001

9. Statistics South Africa. Cataloguing-in-Publication (CIP) Data Census 2011. Methodology and highlights of key results. http://www.statssa.gov.za/census/census_2011/census_products/ Census_2011_Methodology_and_Highlights_of_key_results.pdf (accessed 5 September 2017).

10. Gebremedhn EG, Nagartnam VB. Audit on preoperative fasting of elective surgical patients in an African academic medical center. World J Surg 2014;38(9):2200-2204. https://doi.org/10.1007/s00268014-2582-3

11. Cestonaro T, Madalozzo Schieferdecker ME, Thieme RD, Neto Cardoso J, Ligocki Campos AC. The reality of the surgical fasting time in the era of the ERAS protocol. Nutr Hosp 2014;29(2):437-443. https://doi.org/10.3305/nh.2014.29.2.7025

12. Luttikhold J, Oosting A, van den Braak CC, et al. Preservation of the gut by preoperative carbohydrate loading improves postoperative food intake. Clin Nutr 2013;32(4):556-561. https://doi.org/10.1016/j. clnu.2012.11.004

13. Yagci G, Can MF, Ozturk E, et al. Effects of preoperative carbohydrate loading on glucose metabolism and gastric contents in patients undergoing moderate surgery: A randomized, controlled tial. Nutrition 2008;24(3):212-216. https://doi.org/10.1016/j.nut.2007.11.003

14. Smith MD, McCall J, Plank L, Herbison GP, Soop M, Nygren J. Preoperative carbohydrate treatment for enhancing recovery after elective surgery. Cochrane Database Syst Rev 2014;(8):CD009161. https:// doi.org/10.1002/14651858.CD009161.pub2 